\title{
The Development of Lossless Data Compression Technology for Remote Sensing Applications ${ }^{1}$
}

\author{
Pen-Shu Yeh and Warner H. Miller \\ Goddard Space Flight Center, Code 738.3 \\ Greenbelt, MD 20771 \\ T: 301.286.4477 F:301.286.1751 EMail:psyeh@psy.gsfc.nasa.gov \\ T:301.286.8183 F:301.286.1751 EMail:whmiller@gsfcmail.nasa.gov
}

Lossless data compression has been studied for many NASA missions to achieve the benefit of increased science return; reduced onboard memory requirement, station contact time and communication bandwidth. This paper first addresses the requirement for onboard applications and provides rational for the selection of the Rice algorithm among other available techniques. A toplevel description of the Rice algorithm will be given, along with some new capabilities already implemented in both software and hardware VLSI forms. The paper then addresses systems issues important for onboard implementation including sensor calibration, error propagation and data packetization. The latter part of the paper provides several case study examples drawn from a broad spectrum of science instruments including the thematic mapper, $x$-ray telescope, gammaray spectrometer, acousto-optical spectrometer.

\section{INTRODUCTION}

With the development of new advanced instruments for remote sensing applications, sensor data will be generated at a rate that not only requires increased onboard processing, storage capability, but imposes demands on the communication link and ground data management system. Data compression provides a viable means to alleviate these demands. Two types of data compression have been studied by many researchers in the area of information theory: a lossless technique that guarantees full reconstruction of the data, and a lossy technique which generally gives higher data compaction ratio but incurs distortion in the reconstructed data. To satisfy the many science disciplines NASA supports, lossless data compression becomes the priority for technology development in this area.

To implement a data compression technique on the spacecraft, several criteria are considered:

1. The algorithm has to adapt to the changes in data to maximize performance.

2. It can be easily implemented with few processing steps, small memory and little power.

3. It can be easily interfaced with a packetized data system without performance degradation. There exist a few well known lossless compression techniques including Huffman code, arithmetic code, Ziv-Lempel algorithm and variants of each. After extensive study and performance comparison on the same test image data set (Venbrux, 92)(Yeh, 91, 93), the Rice algorithm originated at Jet Propulsion Laboratories (Rice, 79 ) is selected for implementation.

1. Part of the paper is taken from NASA Technical Paper 3441, "Application Guide for Universal Source Encoding for Space," by the authors, Dec. 1993 and was presented in the International Geoscience and Remote Sensing Symposium, 94. 
The Rice algorithm is essentially a set of Huffman codes organized in a structure that does not require lookup tables. The set of the Huffman codes can be easily extended to the information range of the science data. It is adaptive to the changes in the statistics of the data, and can be easily implemented. The structure of the algorithm also permits simple interface to data packetization scheme without having to carry side information across packet boundary. Therefore its performance is file size independent.

In 1991, a hardware engineering model was built in an Application Specific Integrated Circuit (ASIC) for proof of concept. This particular chip set was named as Universal Source Encoder/ Universal Source Decoder (USE/USD) (Venbrux, 92). Later, it was redesigned with several additional capabilities and implemented in Very Large Scale Integration (VLSI) circuits using gate arrays suitable for space missions. The flight circuit is referred to as Universal Source Encoder for Space (USES). The fabricated USES chip is capable of processing data up to $20 \mathrm{Msamples} / \mathrm{sec}-$ ond and will take data of quantization from 4-bit to 15-bit (MRC, 93).

A description of the Rice algorithm will be given in the next section, followed by systems issues and case study examples on remote sensing data either acquired from launched spacecrafts or simulated for future missions.

\section{THE RICE ALGORITHM ARCHITECTURE}

A block diagram of the architecture of the Rice algorithm (Rice, 91, 93) is given in Figure 1. It consists of a preprocessor to decorrelate data samples and subsequently map them into symbols suitable for the following stage of entropy coding. The entropy coding module is a collection of options operating in parallel over a large entropy range. The option yielding the least number of coding bits will be selected. This selection is performed over a block of $J$ samples to achieve adaptability to scene statistics. An Identification (ID) bit pattern is used to identify the option selected for each block of $\mathrm{J}$ input data.

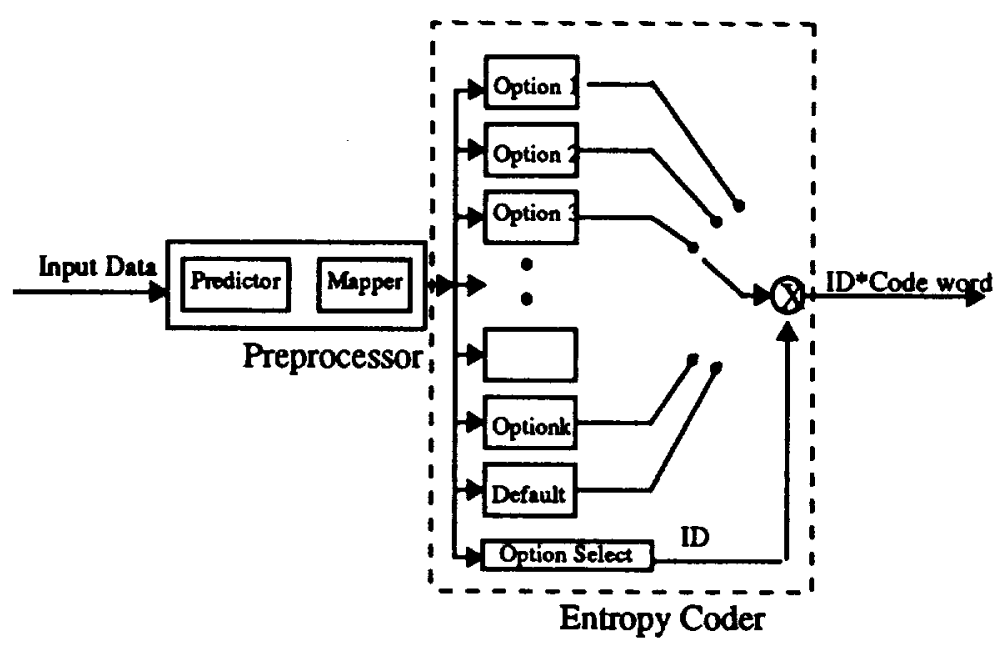

Figure 1. The encoder architecture. 


\section{The Preprocessor}

The predictor in the preprocessor can be as simple as a first order predictor using previous sample, or other higher order predictors. To maintain the pipeline processing in the hardware, only a few predictor types are implemented, these include: a 1D predictor, a 2D predictor, a multispectral predictor and a user-supplied external predictor.

The function of the predictive coder is to decorrelate the incoming data stream by taking the difference between data symbols. The mapper takes these difference values, both positive and negative, and orders them, based on predictive values, sequentially into positive integers.

\section{Entropy Coder}

Most of the options in the entropy coder are called "sample-split options". These options take a block of J preprocessed data samples, split off the $k$ least significant bits, and code the remaining higher order bits with a simple comma code before appending the split-off bits to the coded data stream. Each sample-split option in the Rice algorithm is optimal in an entropy range about one bit/sample (Yeh, 93); only the one yielding the least amount of coding bits will be chosen and identified for a J-sample data block by the option select logic. This assures that the block will be coded with one of the available Huffman codes, whose performance is better than other available options on the same block of data. The $k=0$ option is optimal in the entropy range of $1.5-2.5 \mathrm{bit} /$ sample; the $k=1$ option is optimal in the range of $2.5-3.5 \mathrm{bit} / \mathrm{sample}$, and so on for other $k$ values.

To improve the performance below $1 \mathrm{bi} / \mathrm{sample}$, a new option is devised and included in the full set of options implemented in VLSI. This new option is particularly efficient over data with very low entropy values.

The default option is an option not to use any of the split-sample options or the low-entropy option. It bounds the performance of the algorithm by simply passing through the preprocessed block of data through the encoder without alteration but with an appended identifier.

\section{SYSTEMS ISSUES}

Several systems issues related to embedding data compression scheme onboard a spacecraft should be addressed. These include the relation between the focal-plane array arrangement and the data sampling/prediction direction, the subsequent data packetizing scheme and how it relates to error propagation in case of bit error incurred in the communication channel and how the packetization may affect compression performance.

\section{Sensor Calibration}

Advanced imaging instruments and spectrometers often use arrays of individual detectors arranged in a 1D or 2D configuration; one example is the Charge Coupled Device (CCD). These individual detectors tend to have slight differences in response to the same input photon intensity. For instance, CCDs usually have a different gain and dark current value for each individual detector element. It is important to have the sensor well calibrated so that the data reflects the actual signals received by the sensor. Simulations have shown that for CCD types of sensors, gain and dark current variations as small as $0.2 \%$ of the full dynamic range can render the prediction 
scheme less effective for data compression. Besides calibration, in order to maximize the compression gain, the 1D prediction scheme will be much more effective when data acquired on one detector element is used as the predictor for data acquired on the same detector whose characteristics are stationary, in general, over the data collection period.

\section{Error Propagation}

User acceptability to distortion resulting from channel bit errors is mission dependent, and is strongly a function of received Signal-to-Noise-Ratio (SNR), the data format, the error detection and correction technique and the percent of distorted data that is tolerable. A major concern in using data compression is the possibility of error propagation in the event of a single bit error in the compressed data stream. During the decompression process, a single bit error can lead to a reconstruction error over extended runs of data points. A general approach to minimizing this effect is to provide a very clean channel by using error detection/correction scheme. For compressed data stream, this still does not prevent error propagation, if it occurs, across a decompressed scanline. Further protection can be achieved by using packet data structure in conjunction with a properly chosen error correction scheme as advocated in the Consultative Committee on Space Data Systems (CCSDS) Blue Book (CCSDS, 89). Using this scheme, decompression error resulting from bit-error will then be contained in a packet for compression algorithms that do not carry side information across packet boundary.

\section{Packetization and Compression Performance}

Packetization is used not only as a means to contain bit error locally as just mentioned, it is a logical way to facilitate the transport of variable length bit string as a result of entropy coding. The Rice algorithm chooses an option for every block of samples, its performance is optimized within this block and there is no need to pass side information or statistics across packet boundary. There exist other compression techniques whose performance depends on establishing long-term statistics in a file. These schemes will give good compression performance for a large file and poor performance for a relatively smaller file. When packetization is used in conjunction with these algorithms to prevent error propagation, one would expect better compression performance for a larger packet. The drawback is that the loss of data caused by bit error may be intolerable.

\section{CASE STUDY EXAMPLES}

This section contains several compression study results for several different instruments. The compression performance is expressed as Compression Ratio (CR). It is defined as the ratio of the quantization level in bits to the average code word length, also in bits. It should be noted that the CR value is data dependent and can vary from one test data set to the next.

\section{Landsat Thematic Mapper}

Mission Purpose: The Landsat program was initiated for the study of Earth's surface and resources. Landsat-1, 2, and 3 were launched between 1972 and 1978. Landsat- 4 was launched in 1982, and Landsat-5 in 1984.

Landsat Thematic Mapper (TM) on Landsat-4 and 5: The TM data represent typical land observation data. An image acquired on Landsat- 4 at $30 \mathrm{~m}$ ground resolution for band 1 in the wavelength region of $0.45-0.52 \mu \mathrm{m}$ is shown in Figure 2. This 8-bit 512x512 image was taken over Sierra Nevada in California. 
Compression Study: Using a 1D predictor in the horizontal direction, setting a block size of 16 samples and inserting one reference per every image line, the lossless compression gives a compression ratio at 1.83 for the 8 -bit image.

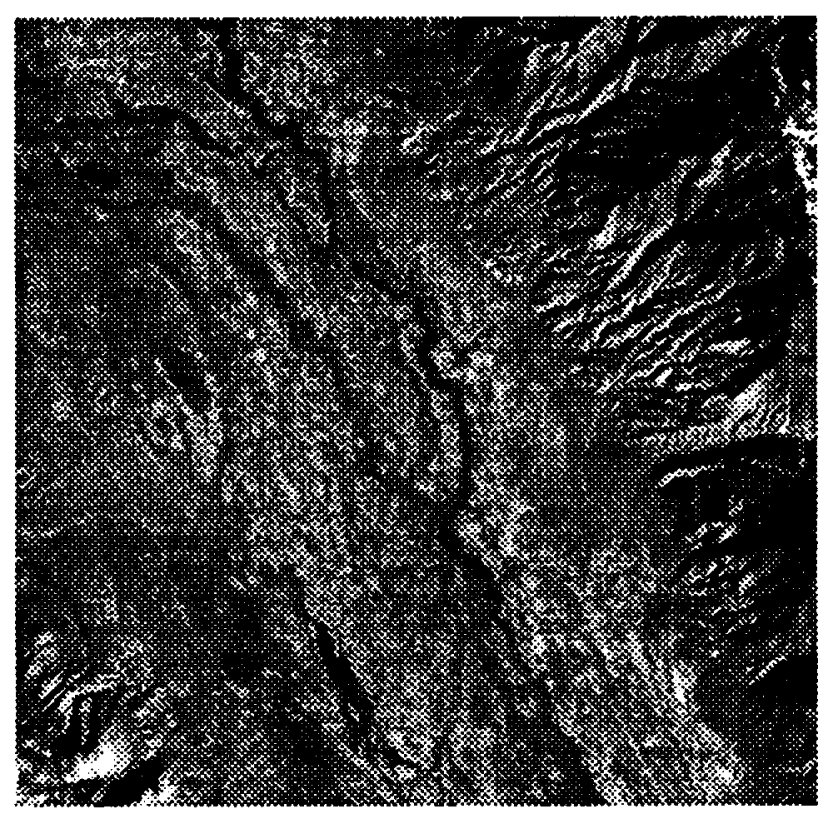

Figure 2. Thematic Mapper image

\section{Soft X-ray Telescope (SXT) on Solar-A Mission}

Mission Purpose: The Solar-A mission, renamed as Yohkoh mission after its successful launch in August, 1991, is dedicated to the study of solar flares, especially of high-energy phenomena observed in the $\mathrm{X}$ - and gamma-ray ranges.

Soft X-ray Telescope (SXT): The instrument detects X-ray in the wavelength range of 3-60 Angstrom. It uses a 1024x1024 CCD detector array to cover the whole Solar disk. Data acquired from the CCD is of 12-bit quantization and is processed on board to provide 8-bit telemetry data. The image in Figure 3 is an averaged image of size 512×512 with dynamic range up to 15 bits in floating point format as a result of further ground processing.

Compression Study: The test image is first rounded to the nearest integer. Then a 1D predictor is applied to this seemingly high-contrast image. A compression ratio of 4.69 is achieved. 


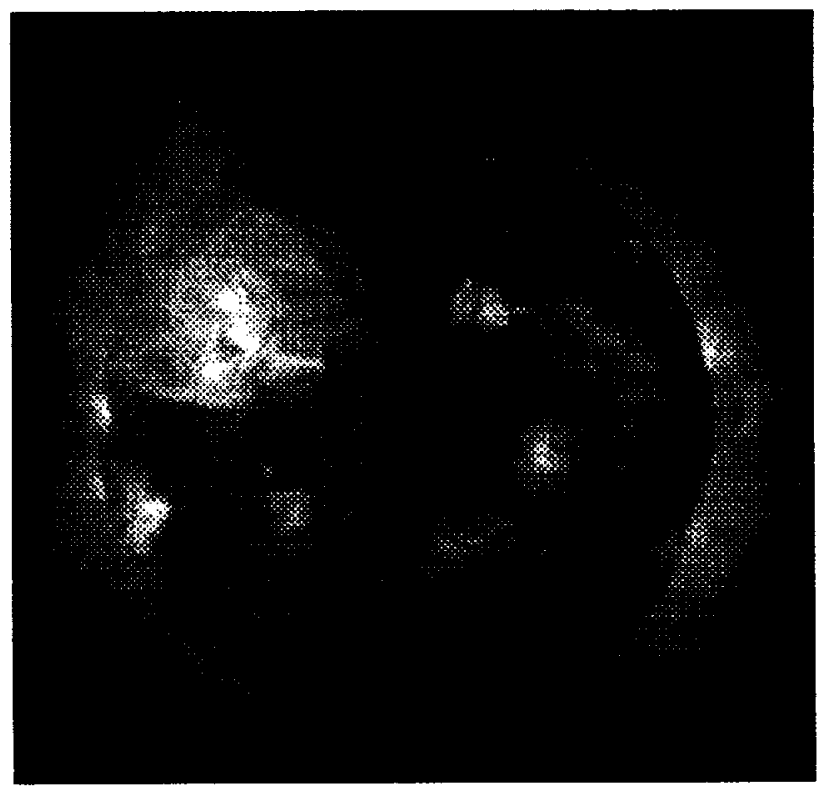

Figure 3. Solar-A X-ray image

\section{Acousto-Optical Spectrometer (AOS) on Submillimeter Wave Astronomy Satellite (SWAS)}

Mission Purpose: The Submillimeter Wave Astronomy Satellite (SWAS) is a Small Explorer (SMEX) mission, scheduled for launch in the summer of 1995 aboard a Pegasus launcher. The objective of the SWAS is to study the energy balance and physical conditions of the molecular clouds in the Galaxy by observing the radio-wave spectrum specific to certain molecules.

Acousto-Optical Spectrometer: The AOS utilizes a Bragg cell to convert the radio frequency energy from the SWAS submillimeter receiver into an acoustic wave, which then diffracts a laser beam onto a CCD array. The sensor has 1450 elements with 16-bit readout. A typical spectrum is shown in Figure 4(a). An expanded view of a portion of two spectral traces is given in Figure 4(b). Because of the detector nonuniformity, the difference in the Analog-to-Digital Converter (ADC) gain between even-odd channels, and effects caused by temperature variations, the spectra have nonuniform offset values between traces, in addition to the saw-tooth-shaped variation between samples within a trace. Because of limited available onboard memory, a compression ratio of over $2: 1$ is required for this mission

Compression Study: 1D prediction between samples is ineffective when the odd and even channels have different ADC gains. Using the multispectral predictor mode, the achievable CR is 2.32. 


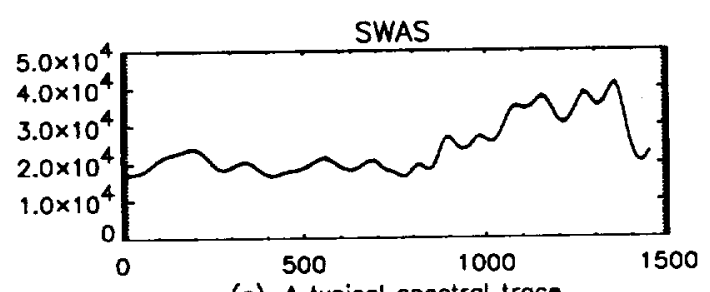

(o). A typical spectral trace

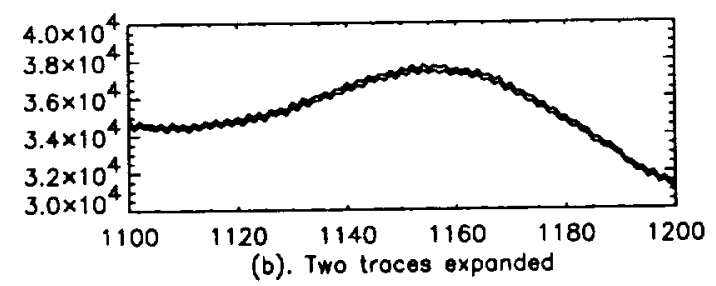

Figure 4. AOS radio wave spectrum

\section{Gamma-Ray Spectrometer on Mars Observer}

Mission Purpose: The Mars Observer was launched in September 1992. The Observer will collect data through several instruments to help the scientists understand the Martian surface, atmospheric properties and the interactions between the various elements involved. In the summer of 93, contact with the spacecraft was lost.

Gamma-Ray Spectrometer (GRS): The spectrometer uses a high-purity germanium detector for gamma rays. The flight spectrum is collected over sixteen thousand channels. The total energy range of a spectrum extends from $0.2 \mathrm{Mev}$ to $10 \mathrm{Mev}$. Typical spectra for a 5 -second and a 50second collection time are given in Figure 5. These spectra show the random nature of the count. The spectral count dynamic range is 8-bit.

Compression Study: The achievable compression depends on the channel collection time. At 5second collection time CR is over 20 and it decreases as collection increases. At 20-second collection time, CR is over 10.
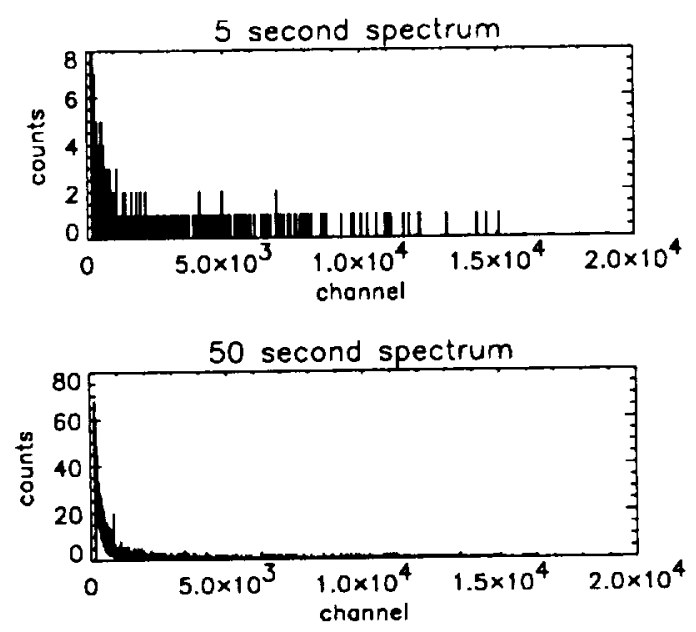

Figure 5. Gamma-ray spectrum 


\section{CONCLUSION}

A lossless data compression technology has been successfully developed for remote sensing applications. This technology is based on the enhanced Rice algorithm. The performance of the algorithm has been established through analysis and simulation. Hardware in VLSI form as well as software are currently available for space flight missions. Over a dozen case studies have been performed on post-flight data and several new missions have adopted the technology for onboard implementation.

\section{REFERENCES}

CCSDS, "Advanced Orbiting Systems: Networks and Data Links: Architectural Specification," CCSDS 701.0-B-1 Blue Book, Oct. 1989. Available from CCSDS Secretariat, Communications and Data systems Division (Code-TS), National Aeronautics and Space Administration, Washington, DC 20546, USA.

MRC, "Universal Source Encoder for Space - USES," Preliminary Product Specification, Version 2.0, Microelectronics Research Center, University of New Mexico, 1993.

Rice, Röbert F., "Some practical universal noiseless coding techniques," JPL Publication 79-22, 1979. Available from author, JPL, 4800 Oak Grove Drive, Pasadena, CA. 91109, USA.

Rice, Robert F., Pen-Shu Yeh and Warner H. Miller, "Algorithms for a Very High Speed Universal Noiseless Coding Module," JPL Publication 91-1, 1991. A shortened version "Algorithms for High-Speed Universal Noiseless Coding," is published in the Proceedings of the AIAA Computing in Aerospace 9 Conference, San Diego, CA, Oct. 19-21, 1993

Venbrux, Jack, Pen-Shu Yeh and Muye N. Liu, "A VLSI Chip Set for High-Speed Lossless Data Compression," IEEE Trans. on Circuits and Systems for Video Technology, Vol. 2, No. 4, Dec. 1992.

Yeh, Pen-Shu, Robert F. Rice and Warner H. Miller, "On the Optimality of Code Options for a Universal Noiseless Coder," Revised version, JPL Publication 91-2, 1991. A shortened version "On the Optimality of a Universal Noiseless Coder," is published in the Proceedings of the AIAA Computing in Aerospace 9 Conference, San Diego, CA, Oct. 19-21, 1993. 